\title{
$\begin{array}{ll}\text { Research Square } & \text { Preprints are preliminary reports that have not undergone peer review. } \\ \text { They should not be considered conclusive, used to inform clinical practice, } & \text { or referenced by the media as validated information. }\end{array}$
}

\section{Efficacy and safety of wait and see strategy versus surgery for rectal cancer with cCR/near-cCR response after neoadjuvant chemoradiotherapy: a meta-analysis}

\section{Guo-hua Zhao}

cancer hospital of China medical university, Liaoning cancer hospital and institute

\section{Li Deng}

Liaoning University of Traditional Chinese Medicine Affiliated Hospital

\section{Dong-man Ye}

cancer hospital of China medical university, Liaoning cancer hospital and institute

\section{Wen-hui Wang}

cancer hospital of China medical university, Liaoning cancer hospital and institute

\section{Yan Yan}

cancer hospital of China medical university, Liaoning cancer hospital and institute tao yu ( $\nabla$ yutao611482@sina.com )

Cancer hospital of China medical university, Liaoning cancer hospital and insititute

\section{Research}

Keywords: wait and see, radical surgery, local excision, cCR, near-cCR, meta-analysis

Posted Date: March 30th, 2020

DOI: https://doi.org/10.21203/rs.2.24658/v2

License: (c) (i) This work is licensed under a Creative Commons Attribution 4.0 International License.

Read Full License 


\section{Abstract}

Background: To evaluate the efficacy and safety between wait and see strategy (WS) and surgery of rectal cancer patients with cCR/near-cCR response after neoadjuvant chemoradiotherapy.

Methods: We searched PubMed, Cochrane Library, CNKI(China National Knowledge Infrastructure) and Wanfang databases to compare wait and see strategy with surgery for rectal cancer with cCR/near-cCR response after neoadjuvant chemoradiotherapy up to January 2020. We collected the data of local recurrence, distant metastasis, cancer related death, overall survival and diseasr-free survival and compared the advantages and disadvantages of the two groups.

Results: 14 English studies with 3932 patients were included. There were 700 patients in WS group and 3232 patients in surgical group. WS group had higher local recurrence rate than surgery group(OR:3.55, $95 \% \mathrm{Cl}: 2.35$ to $5.36, P<0.001)$. WS group had better 2-year DFS(OR:0.74, $95 \% \mathrm{Cl}: 0.56$ to $0.96, P=0.03$ ) and 2-year OS (OR:0.38, $95 \% \mathrm{Cl}: 0.28$ to $0.52, P<0.001)$ than surgery group. Subgroup analysis of WS group and radical surgery group also obtained the similar results. Eastern studies also supported the conclusion. There was no significant difference of other data between the two groups.

Conslusion: Compared with surgery group, WS group would increase the risk of local recurrence rate, but WS group had better 2-year DFS and OS than surgery group. However, WS group did not increase the possibility of distant metastasis and cancer related death of the patients.

\section{Background}

Colorectal cancer is a serous threat to human health, mid-low rectal cancer accounts for about

70 percent of colorectal cancer[1,2]. Radical surgery remains the main treatment for mid-low rectal cancer, but 10-20 percent of patients still have local recurrence after radical surgery[3,4]. However, neoadjuvant chemoradiotherapy has reduced the local recurrence rate of patients and preserved the anus. Approximately 20 percent of the rectal cancer patients have a good response to neoadjuvant chemoradiotherapy and the tumor disappear completely. This phenomenon is called clinical complete response(CCR)[5]. The stringent definition of CCR was proposed by Mass in 2012, and it contained five diagnostic criteria. The diagnostic criteria of CCR included no residual tumor and white scar in endoscopy, negative biopsies from the white scar, no palpable tumor with digital rectal exam (DRE), no suspicious lymph nodes and substantial downsizing with no residual tumor or residual fibrosis in MRI. And the diagnostic criteria of near-cCR included small residual erythematous ulcer or irregular wall thickening in endoscopy, dyspalstic changes by histopathology, small superficial soft irregularity in digital rectal exam (DRE), obvious downstaging of lymph nodes without malignant enhancement pattern(remaining nodes $\geq 5 \mathrm{~mm}$ ) and obvious downstaging with residual fibrosis but irregular aspect in MRI. In our opinion, the most critical point in adopting the WS strategy is no residual tumor in endoscopy and no suspicious lymph nodes or residual tumor in MRI. 
In 2004, Habr-Gama published the results of rectal cancer patients with cCR status after neoadjuvant chemoradiotherapy. She first proposed Wait and see(WS) strategy of treating rectal cancer patients with cCR status. She pointed out that Wait and see(WS) strategy could achieve similar clinical effects as surgery[6]. Although there were several meta-analysis about this topic, such as Li's research, Dossa's research and so on[7-8]. But our study included more researches and more patients to explore the efficacy and safety of wait and see strategy versus surgery for rectal cancer with $\mathrm{CCR} /$ near-cCR response after neoadjuvant chemoradiotherapy. We also performed a subgroup analysis to compare the efficacy and safety of WS strategy versus radical surgery and WS strategy versus local resection.

\section{Methods}

\section{Literature Search}

We carried out the meta-analysis by using the PRISMA guildlines. The details of PICOS were as follows: Population: rectal cancer patients with $\mathrm{cCR} /$ near-cCR response after neoadjuvant chemoradiotherapy; Intervention: wait and see strategy or surgery; Comparator: long-term outcomes contained local recurrence, distant metastasis, cancer related death, disease-free survival(DFS) and overall survival(OS); Outcomes: long-term outcomes were analyzed and compared. Continuous data were evaluated by the standardized mean differences (SMD) with 95\% confidence intervals (95\% Cls) and dichotomous outcomes were evaluated by relative risks (ORs or RRs) with $95 \%$ Cls.

We searched Cochrane Library, PubMed, CNKI(China National Knowledge Infrastructure) and Wanfang databases(up to January 2020). Articles about wait and see versus surgery after neoadjuvant chemoradiotherapy for rectal cancer were collected. In order to avoid missing the useful articles, we would expand the scope of search terms and find out articles for the purpose by manual screening. The search terms were "wait and see" or "nonoperative management" and "neoadjuvant chemoradiotherapy" and "rectal cancer". The details were shown in the additional file 1.

\section{Inclusion and exclusion criteria}

There were 4 inclusion criteria. 1.pathological and long-term outcomes were compared

between wait and see versus surgery for rectal cancer with CCR/near-cCR response after neoadjuvant chemoradiotherapy; 2.surgery included radical surgery and local excision; 3.cCR or near-cCR response after neoadjuvant chemoradiotherapy (criteria for ccR and near-cCR were mentioned above) [9]; 4.RCT(Randomized Controlled Trial), RCNTs(retrospective comparative non-randomized studies), PCNTs(prospective comparative non-randomized studies), cohort studies or case-control studies.

There were 3 exclusion criteria. 1. studies with no valuable outcome; 2. patients were not well grouped or confusing group was not suitable for the purpose of the article; 3 . bad clinical response of rectal cancer after neoadjuvant chemoradiotherapy. Bad clinical response included larger residual tumor in endoscopy and DRE, positive biopsies, larger tumor and lymph nodes in MRI. 


\section{Data extraction and Quality control}

By using the Newcastle-Ottawa Scale (NOS) guildlines, two reviewers searched the literatures (GHZ and DMY) independently [10]. We collected the useful data and showed in table 1 and table 2. Table 1 contained study characteristics, such as: the first author, clinical stage, publication year, country, age, study size, study type, study quality, neoadjuvant therapy plan, evaluation time, follow-up time, radical surgery type, $\mathrm{T}$ stage, $\mathrm{N}$ stage and NOS score. Table 2 contained long-term outcomes, such as: local recurrence, distant metastasis, cancer related death, disease free survival, overall survival. A third reviewer had the final decision power to resolve the disagreements of the study. We tried to contact the authors with missing data, but did not get any relevant data.

\section{Statistical analysis}

We used Revman 5.0 to carry out the meta-analysis. Continuous data was evaluated by the standardized mean differences (SMD) with 95\% confidence intervals (95\% Cls) and dichotomous data was evaluated by relative risks (ORs or RRs) with $95 \%$ Cls. Heterogeneity and publication bias were estimated by $P$ statistic and funnel plots separately. When huge heterogeneity with $R \nabla 50 \%$, we used random effects models to analyze the data. And we used the fixed effects model to analyze the data with little heterogeneity $(R \leq 50 \%)$.

\section{Results}

\section{Study selection}

Duplicated records were deleted. We deleted 1843 studies after reading the titles and abstracts carefully. Deleted studies were due to not rectal cancer $(n=527)$, confusing group $(n=851)$ and insufficient data( $(\mathrm{n}=455)$. After we read the remaining studies carefully, 14 English studies with 3932 patients were included [6,11-23]. There were 3 patients in the WS group and 1 patient in the surgical group with Stage IV. There are 700 patients in WS group and 3232 patients in surgical group. The clinical stages of included patients are邓to IV stage. LE groups did not included patient with stage 0 and/or Stage IV. Surgical group divided into radical surgical group $(n=3140)$ and LE group (local excision group, $n=92$ ) (Table 1). Two groups had similar patient baseline with no significant difference. There were 6 Eastern studies and 8 Western studies in the meta-analysis. The Western research includes European, American, and Latin American research, while the Eastern research mainly includes Asian research. Martens, Yeom, Lin and Lai reported local resection for rectal cancer with cCR/near-cCR response after neoadjuvant chemoradiotherapy. Rectal cancer patients which performed radical surgery and local excision were put together for analysis in Lin's research, the patient outcome of two surgery subgroups was not described separately. So there were only 92 patients data could be collected. Martens described near-cCR in his study, there were patients with 39 near-cCR and 61 patients with $\mathrm{CCR}$ in both groups. There is no possibility in patient profiles among WS, RS and LE groups. There was no statistical difference in the baseline data of the included patients for each article. 
Most of the studies have used the above five criteria to determine whether patients have achieved cCR or near-cCR[16-23]. Araujo used endoscopy, DRE and MRI to confirm cCR[11]. Ayloor used endoscopy, DRE, $\mathrm{CT}$ and ultrasound to confirm CCR[12]. Dalton used endoscopy and MRI to confirm cCR[13]. Habr used endoscopy, DRE and CT to confirm cCR[6]. Lai used endoscopy and MRI to confirm cCR[14]. Lee used DRE and MRI to confirm cCR[15]. The detail was shown in table 1.

\section{Quality assessment}

We evaluated the quality of the included studies using NOS assessment scale (Newcastle Ottawa Quality Assessment Scale). The qualities of included studies were divided into three levels: low level $(1<$ scores $\leq 3)$, moderate level $(4<$ scores $\leq 6)$ and high level $(7 \leq$ scores $\leq 9)$. We included 14 studies in our study (5 RCNT and 9 PNCT). No relevant RCTs was been found during the database search. Seven studies were of moderate quality and seven studies of high quality. The quality scores and study type were shown in table 1.

\section{WS group versus Surgery group}

\section{Local recurrence, Distant metastasis and Cancer related death}

12 studies reported clinical data on local recurrence, WS group had higher recurrence rate than surgery group in the fixed-effects model (OR:3.55, 95\% confidence interval [Cl]:2.35 to $5.36, P<0.001$, chi $^{2}=9.9$, $P=0.54, P=0 \%$, Fig 2a). Both Western (OR:3.14, 95\% Cl: 1.31 to 7.52, $P=0.01$, chi $^{2}=8.14, P=0.15, P=39 \%$, Fig 2a) and Eastern studies (OR:3.74, 95\% Cl:2.39 to 5.85, $P<0.01, \mathrm{chi}^{2}=1.39, P=0.92, P=0 \%$, Fig 2a) had the same results in local recurrence. Distant metastasis (OR:0.94, 95\% Cl:0.68 to $1.29, P=0.69$, chi $^{2}=14.76$, $P=0.19, P=25 \%$, Fig 2b) and cancer related death (OR:0.77, 95\% Cl:0.32 to $1.84, P=0.56, \mathrm{chi}^{2}=5.54, P=0.24$, $R=28 \%$, Fig 2 c)were similar between two groups in the fixed-effects model with little heterogeneity.

\section{WS group versus Surgery group}

\section{2-year DFS, 2-year OS, 5-year DFS and 5-year OS}

10 studies reported 2-year DFS and WS group had better 2-year DFS than surgery group in the fixedeffects model with little heterogeneity (OR:0.74, $95 \% \mathrm{Cl}: 0.56$ to $0.96, P=0.03$, chi $^{2}=9.01, P=0.44, R=0 \%$, Fig 3a). 9 studies reported 2-year OS and WS group had better 2-year OS than surgery group in the fixedeffects model with little heterogeneity (OR:0.38, $95 \% \mathrm{Cl}: 0.28$ to $0.52, P<0.001$, chi $^{2}=13.89, P=0.05, P=50 \%$, Fig $3 b)$. Eastern research rather than Western research indicated 2-year DFS and 2-year OS were better in WS group than in surgical group. 5-year DFS (OR:0.46, 95\% Cl:0.19 to $1.12, P=0.09$, chi $^{2}=20.55, P=0.001$, $P=76 \%$, Fig 3c) and 5-year OS (OR:0.79, 95\% Cl:0.29 to 2.14, $P=0.65$, chi $^{2}=11.19, P=0.05, P=55 \%$, Fig $3 d)$ were similar in both groups in the random-effects model with huge heterogeneity.

\section{Subgroup analysis}




\section{WS group versus Radical surgery group}

\section{Local recurrence and Distant metastasis}

11 studies reported clinical data on local recurrence, WS group had higher recurrence rate than radical surgery group in the fixed-effects model (OR:4.71, $95 \% \mathrm{Cl}: 3.07$ to $7.21, P<0.001$, $\mathrm{chi}^{2}=4.1, P=0.94,{ }^{2}=0 \%$, Fig 4a). Both Western (OR:7.47, 95\% Cl:2.17 to 25.64, $P=0.001$, chi $^{2}=2.13, P=0.71, P=0 \%$, Fig $4 a$ ) and Eastern studies (OR:4.18, 95\% Cl:2.65 to $6.58, P<0.001$, chi $^{2}=1.26, P=0.94, P=0 \%$, Fig $4 a$ ) had the same results in local recurrence. WS group had similar distant metastasis rate with radical surgery group in the fixed-effects model with high heterogeneity (OR:0.97, $95 \% \mathrm{Cl}: 0.70$ to $1.35, P=0.87$, $\mathrm{chi}^{2}=14.57, P=0.15$, $R=31 \%$, Fig $4 \mathrm{~b})$.

\section{WS group versus local excision group}

\section{Local recurrence and Distant metastasis}

Similar distant metastasis rate was in two groups in the fixed-effects model with little heterogeneity(OR:1.10,95\% Cl:0.46 to 2.63, $P=0.84$, $\mathrm{chi}^{2}=2.5, P=0.29, P=20 \%$, Fig $4 \mathrm{c}$ ). 3 studies reported clinical data on local recurrence, local recurrence was similar in two groups in the fixed-effects model (OR:0.78, $95 \% \mathrm{Cl}: 0.26$ to $2.36, P=0.66, \mathrm{chi}^{2}=3.05, P=0.22, P=34 \%$, Fig $4 \mathrm{~d}$ ).

\section{WS group versus Radical surgery group}

\section{2-year DFS and 5-year DFS}

10 studies reported 2-year DFS and WS group had better 2-year DFS than surgery group in the fixedeffects model with little heterogeneity (OR:0.73, $95 \% \mathrm{Cl}: 0.56$ to $0.95, P=0.02$, chi $^{2}=9.48, P=0.39, R=5 \%$, Fig $5 a)$. Eastern research rather than Weatern research had the same result (OR:0.70, 95\% Cl:0.51 to 0.96, $P=0.03$, chi $^{2}=0.75, P=0.69, R=0 \%$, Fig 5a). 5 -year DFS was similar in both groups in the random-effects model with huge heterogeneity (OR:0.44, 95\% Cl:0.18 to $1.12, P=0.09$, $\mathrm{chi}^{2}=21.86, P<0.001, P=77 \%$, Fig $5 b)$.

\section{WS group versus Local excision group}

\section{3-year DFS}

2 studies indicated WS group and local excision group had the similar 3-year DFS in the fixed-effects model (OR:0.35, 95\% Cl:0.09 to 1.29, $P=0.11, \mathrm{chi}^{2}=0.33, P=0.56, P=0 \%$, Fig $5 \mathrm{c}$ ).

\section{WS group versus Radical surgery group(CCR)}

Local recurrence, Distant metastasis and Cancer related death 
Due to only one research about near-cCR(Martens), the result of local recurrence and distant metastasis were the same to fig $4 \mathrm{a}$ and fig $4 \mathrm{~b}$ (WS group versus Radical surgery group). The result of cancer related death was the same to fig 2c (WS group versus Radical surgery group).

\section{WS group versus Radical surgery group(CCR)}

\section{2-year DFS, 2-year OS, 5-year DFS and 5-year OS}

Due to no available information of the only one near-cCR research (Martens), the result of 2-year DFS, 2year OS, 5-year DFS and 5-year OS were the same to fig 3a, fig 3b, fig 3c and fig 3d(WS group versus Surgery group).

\section{WS group versus local excision group(cCR)}

\section{Local recurrence and Distant metastasis}

2 studies indicated WS group and local excision group had the similar local recurrence (OR:1.46, 95\% Cl:0.49 to $4.35, P=0.5, \mathrm{chi}^{2}=1.69, P=0.19, R=41 \%$, Fig $5 \mathrm{~d}$ ) and distant metastasis (OR: $1.24,95 \% \mathrm{Cl}: 0.33$ to 4.69, $P=0.75, \mathrm{chi}^{2}=0.94, P=0.33, P=0 \%$, Fig $\left.5 e\right)$ in the fixed-effects model.

There was one research with cCR which perform local excision, it can not be studied by meta-analysis. There was one research with near-cCR which perform local excision, so it can not be studied by metaanalysis too. Due to the lack of related research about the near-cCR status, there is no large amount of data for meta-analysis.

\section{Publication bias}

In order to determine whether the article had publication bias, we used Revman5.0 software to test the index of distant metastasis rate of the included literature and obtained the funnel plot. The points were evenly distributed in the funnel plot and it indicated no publication bias in the meta-analysis.

\section{Discussion}

Radical surgery of mid-low rectal cancer could bring great trauma to patients and some patients could need to remove their anus. It had a serious impact on patients' physical and mental health[24]. In 2004, Habr-Gama first reported wait and see(non-surgical) treatment strategy for low rectal cancer patients who obtained CCR after chemoradiotherapy. 71 patients which achieved CCR status were only closely followed(wait and see) for an average of 57.3 months. 2 patients had recurrence in the intestinal lumen and 3 patients had distant metastasis. 5-year DFS rate and 5-year OS rate were $92 \%$ and $100 \%$ separately. The other 194 patients without clinical complete response undergo radical surgery. 22 patients had pathologically confirmed $\mathrm{pCR}$ (pathologically complete response). 5-year disease free survival rate and 5year overall survival rate were $83 \%$ and $88 \%$. No significant difference was found between the two groups. After expanding the sample size, Habr-Gama got the same conlusion. Subsequently, many 
studies about CCR had emerged, and they also confirmed the treatment effect of wait and see strategy. With the gradual understanding of CCR patients, people have also turned their attention to the near-cCR patients. Near-cCR patients also accounted for 20-30 percentage of rectal cancer patients after neoadjuvant chemoradiorherapy. Lu et al found that $50 \%$ of ypT1-2 tumors could disappear completely and postoperative pathology was ypT0[25]. Therefore, we also included cCR/near-cCR patients and intended to compare the advantages and disadvantages of the two treatment methods.

In this meta-analysis, we found WS group had higher local recurrence rate than surgery group.

Our research result was similar with Li's research. 64 patients had local recurrence in WS group (565 patients), while 103 patients had local recurrence in surgery group (3117 patients). In subgroup analysis, Eastern and Western research both supported the above conclusion, so was the radical surgery group. Due to few patients in local excision group with incomplete information, no significant difference of local recurrence rate was seen in the LE group of subgroup analysis. There was no significant difference of distant metastasis rate and cancer related death in two groups. No difference was found by subgroup analysis. Dossa and Li also got the same result. Wait and see strategy had some advantages, such as: reducing surgical trauma, improving the quality of life, no raising distant metastasis rate and so on.

WS group had better 2-year DFS and OS than surgery group, Eastern research and radical surgery group indicated the same result in subgroup analysis. WS group with higher local recurrence rate have better DFS and OS than surgery group. We speculated that high sensitivity MRI and colonoscopy help patients to find small tumor lesion and deal with them in time[26,27]. The included articles had salvage therapy for patients with local recurrence and alvage therapy included chemotherapy and surgical treatment. The patients of WS group which had not experienced large surgical trauma could better immunity than surgical group, and they could endure the subsequent treatments. The above factors may lead to a better OS in the WS group than surgical group. Lin's research published in 2018 with 2780 patients, it could cause publication bias and affected the result. 5-year DFS and OS were similar in both groups, this result were consistent with the above two meta-analysis.

In our research, 2-year DFS and OS of Eastern research were better than Western research. We speculated that it was still relevant to lin's study with large sample. The incomplete data of 2-year DFS and OS in some studies could lead to publication bias and affect the result. Unlike radical surgery group, fewer patients in LE group indicated no positive result, we hope more studies about cCR/ Near-cCR related research of the WS group versus surgery group (radical surgery and local excision) would appear[28].

Although Dossa included 23 studies in his research, only 15 had control groups and 5 had specific data of comparison between two groups[8]. He proposed that there was no significant difference in nonregrowth recurrence, cancer-specific mortality, overall survival and disease-free survival. There were 9 studies in Li's research and he reported that two patient groups did not differ in distant metastasis rates or disease-free and overall survival, but the nonsurgical group had a higher risk of 1,2,3, and 5-year local recurrence[7]. Our report also showed higher local recurrence rate in WS group than surgery group. We also reported WS group had better 2-year DFS and 2-year OS than surgery group, it could promote WS 
treatment popular. Subgroup analysis of WS group, radical surgery group, local excision group, Eastern studies and Western studies are also performed.

There were 3 patients in the WS group and 1 patient in the surgical group with Stage IV. The specific situation of patients with metastasis is not specifically described in the article, we speculate that it may be due to new metastases during neoadjuvant therapy, and the malignany is mild so the follow-up treatment was continued. It could cause the bias of the result. More cases with of perfect T3-4 and N1-2 stage are more likely the perform neoadjuvant treatment than T1-2 and N0 stage. We implied some patients with T3-4 and N1-2 stage could obtain CCR after neoadjuvant treatment with better prognosis. Sometimes, T1-2 and N0 stage may be treated directly with surgery and obtain considerable effect. However, the relevant prognosis of patients with $\mathrm{T}$ stage and $\mathrm{N}$ stage were not specified in the article, and we could not use the meta-analysis to know the specific meaning of $\mathrm{T}$ stage and $\mathrm{N}$ stage for CCR or nearcCR. Most patients had achieved $\mathrm{T}$ and $\mathrm{N}$ stage reduction after neoadjuvant therapy, but the available information is too little to make a conclusion of specific treatment method for the patients.

This study might have several limitations. First, 14 studies (5 RCNT and 9 PNCT) with a total of 3932 patients could represent the highest level of evidence due to no RCT was included. Second, Lin's study contained 2/3 patients and incomplete clinical data could affect the results. Third, differences between Eastern and Western population groups could cause potential selection bias. Finally, near-cCR patient inclusion criteria need to be clear. Furthermore, more RCTs of wait and see strategy versus surgery for cCR/near-cCR were necessary.

\section{Conclusion}

In summary, this study had compared the reliability and safety of wait and see strategy versus surgery for rectal cancer with CCR/near-cCR response after neoadjuvant chemoradiotherapy. WS group had higher local recurrence rate than surgery group, but WS group had better 2-year DFS and OS than surgery group. There was no statistical difference in other data. Subgroup analysis also indicated the similar results. With neoadjuvant chemoradiotherpay development and appropriate salvage therapy for local recurrence, wait and see strategy could minimize surgical trauma and preserve anus. Wait and see strategy had the advantages for cCR/near-cCR patient. Therefore, we proposed that Wait and see strategy could be a feasible model for CCR/near-cCR patient.

\section{Declarations}

\section{Acknowledgements}

We thank the Department of General surgery and Medical Imaging of the Cancer Hospital of China Medical University, Liaoning Cancer Hospital and Institute for technical assistance.

\section{Funding}


This work was supported by the Cancer Hospital of China Medical University.

The funding project is the Science and Technology Department of Liaoning Province Fund from the Liaoning Province Natural Science Foundation of China (grant nos.2014225016).

\section{Availability of data and materials}

All data generated or analysed during this study are included in this published article.

\section{Authors' contributions}

All authors participated in the study. GHZ, DMY performed the literature search and the acquisition of data. LD and YY performed data analysis. WHW and TY participated in the interpretation of data and revised the article for important intellectual content. All authors approved the final version of the article.

\section{Ethics approval and consent to participate}

Not applicable

\section{Consent for publication}

Not applicable

\section{Competing interests}

The authors declare that they have no conflicts of interest.

\section{Publisher's Note}

Springer Nature remains neutral with regard to jurisdictional claims in published maps and institutional affiliations.

\section{Abbreviations}

PNCT: prospective non-randomized controlled trial;

RNCT: retrospective non-randomized controlled trial;

5-FU: 5-Fluorouracil;

Cape: Capecitabine;

RS: radical surgery;

TME: total mesorectal excision;

APR: abdominal-perineal resection; 
LAR: Low anterior resection;

Cl: confidence interval;

OR: Odds ratios;

OS: Overall survival;

SMD: Standardized mean difference.

\section{References}

[1] Sakamoto Y, Morohashi H, Yoshida E, et al. Treatment Strategy for Stage $\otimes$ Rectal Cancer. Gan To Kaqaku Ryoho. 2015,42:1313-1515.

[2] Russell MM, Ganz PA, Lopa S, et al. Comparative Effectiveness of Sphincter-Sparing Surgery Versus Abdominoperineal Resection in Rectal Cancer: Patient-Reported Outcomes in National Surgical Adjuvant BrEast and Bowel Project Randomized Trial R-04. Ann Surg. 2015, 261:144-8

[3] F. Dumont, A. Mariani, D. Elias, D, et al. Surgical strategy for low rectal cancers. J Visc Surg. 2015, 152:739-744.

[4] Polanco PM, Mokdad AA, Zhu H, et al. Association of adjuvant chemotherapy with overall survival in patients with rectal cancer and pathologic complete response following neoadjuvant chemotherapy and resection. JAMA oncology. 2018,4:938-943.

[5] Cui Y, Yang X, Shi Z, et al. Radiomics analysis of multiparametric MRI for prediction of pathological complete response to neoadjuvant chemoradiotherapy in locally advanced rectal cancer. Eur Radiol. 2019,29:1211-1220.

[6] Habr-Gama A, Perez RO, Nadalin W, et al. Operative versus nonoperative treatment for stage 0 distal rectal cancer following chemoradiation therapy: long-term results. Ann Surg. 2004, 240:711-717.

[7] Li J, Li L, Yang J, et al. Wait-and-see treatment strategies for rectal cancer patients with clinical complete response after neoadjuvant chemoradiotherapy: a systematic review and meta-analysis. Oncotarget. 2016, 7:44857-44870.

[8] Dossa F, Chesnev TR, Acuna SA, et al. A watch-and-wait approach for locally advanced rectal cancer after a clinical complete response following neoadjuvant chemoradiation: a systematic review and metaanalysis. Lancet Gastroenteol Hepatol. 2017,2:501-513.

[9] Buijo K, Rutkowski A. RE: Long-term Outcome of an Organ Preservation Program After Neoadjuvant Treatment for Rectal Cancer. J Natl Cancer Inst. 2016,108:djw291. 
[10] Wells GA, Shea B, O'Connell D, et al. The Newcastle-Patent Scale (NOS) for assessing the quality of nonrandomised studies in meta- analyses. Ottawa Hospital Research Institute Web Site. http://www ohri ca/ programs/clinical epidemiology/oxford.asp. Accessed October 2017;25.

[11] Arauio RO, Valadão $M$, Borges $D$, et al. Nonoperative management of rectal cancer after chemoradiation opposed to resection after complete clinical response. A comparative study. Eur J Surg Oncol. 2015,41:1456-1463.

[12] Ayloor SR, Kondaveeti SS, Jayanand SB, et al. Complete clinical response to neoadjuvant chemoradiation in rectal cancers: can surgery be avoided? Hepatogastroenterology. 2013, 60):410-414.

[13] Dalton RS, Velineni R, Osborne ME, et al. A single-centre experience of chemoradiotherapy for rectal cancer: is there potential for nonoperative management? Colorectal Dis. 2012, 14:567-571.

[14] Lai CL, Lai MJ, Wu CC, et al. Rectal cancer with complete clinical response after neoadjuvant hemoradiotherapy, surgery, or "watch and wait" Int J Colorectal Dis. 2016, 31):413-419.

[15] Lee SY, Kim CH, Kim YJ, et al. Oncologic Outcomes according to Treatment Strategy in Radiologic Complete Responders after Neoadjuvant Chemoradioation for Rectal Cancer. Oncology. 2015, 89:311318.

[16] Li J, Liu H, Yin J, et al. Wait-and-see or radical surgery for rectal cancer patients with a clinical complete response after neoadjuvant chemoradiotherapy: a cohort study. Oncotarget. 2015, 6::42354261.

[17] Lin GP, Lee KD, Wang JY, et al. Status for clinically complete remission rectal cancer after concomitant chemo-radiotherapy in Taiwan. Asian J Surg. 2018, 41:203-206.

[18] Martens MH, Mass M, Heijnen LA, et al. Long-term outcome of an organ preservation program after neoadjuvant treatment for rectal cancer. J Natl Cancer Inst. 2016,108(12):djw171.

[19] Maas M, Beets-Tan RG, Lambregts DM, et al. Wait-and-see policy for clinical complete responders after chemoradiation for rectal cancer. J Clin Oncol. 2011, 29:4633-4640.

[20] Renehan AG, Malcomson L, Emsley R, et al. Watch-and-wait approach versus surgical resection after chemoradiotherapy for patients with rectal cancer (theOnCoRe project): a propensity-score matched cohort analysis. Lancet Oncol. 2016,17:174-183.

[21] Smith JD, Ruby JA, Goodman KA, et al. Nonoperative management of rectal cancer with complete clinical response after neoadjuvant therapy. Ann Surg. 2012, 256:965-972.

[22] Smith RK, Fry RD, Mahmoud NN, et al. Surveillance after neoadjuvant therapy in advanced rectal cancer with complete clinical response can have comparable outcomes to total mesorectal excision. Int $\mathrm{J}$ Colorectal Dis. 2015,30:769-774. 
[23] Yeom SS, Lee SY, Kim CH, et al. Non-operative treatment outcome for rectal cancer patient with clinical complete response after neoadjuvant chemoradiotherapy. Asian J Surg. 2019,42:823-831.

[24] Oldani A, Cesana G, Uccelli M, et al. Surgical outcomes of rectal resection: our 10 years experience. J Laparoendosc Adv Surg Tech A. 2019,29:820-825.

[25] Lu HT, Liu X, Shi G, et al. A comparative study of the minimal invasive surgery and radical surgery in low rectal cancer with almost-cCR(near-cCR) after neoadjuvant therapy. Modern Oncology. 2019,27:29002904.

[26] Aker M, Ganeshan B, Afaq A, et al. Magnetic resonance texture analysis in identifying complete pathological response to neoadjuvant treatment in locally advanced rectal cancer. Dis Colon Rectum. 2019,62:163-170.

[27] Li SJ, Wang L, Zhang XY, et al. Application value of colonoscopic assessment in "watch and wait" strategy for mid-lower rectal cancer after neoadjuvant chemoradiotherapy. Zhonghua Wei Chang Wai Ke Ze Zhi. 2019,22:648-655.

[28] Rullier E, Rouanet $P$, Tuech JJ, et al. Organ preservation for rectal cancer (GRECCAR 2): a prospective, randomised, open-label, multicentre, phase 3 trial. Lancet. 2017,390:469-479.

\section{Table}

Table 1 Characteristics of the included articles 


\begin{tabular}{|c|c|c|c|c|c|c|c|c|c|c|c|}
\hline & \multirow[t]{2}{*}{ Year } & \multirow[t]{2}{*}{ Country } & \multicolumn{3}{|c|}{ Case } & \multicolumn{3}{|c|}{ Age } & \multirow[t]{2}{*}{ cCR/ near-cCR } & \multirow[t]{2}{*}{ Diagnostic criteria } & \multirow{2}{*}{$\begin{array}{l}\text { Study } \\
\text { design }\end{array}$} \\
\hline & & & WS & $\mathrm{RS}$ & LE & WS & RS & $\mathrm{LE}$ & & & \\
\hline & 2015 & Brazil & 42 & 69 & - & 63.6 & 60.1 & - & cCR & प्राप्रा & PNCT \\
\hline & 2013 & India & 23 & 10 & - & 50 & 55 & - & $\mathrm{cCR}$ & $\mathrm{CO}+\mathrm{CT}$ and ultrasound & RNCT \\
\hline & 2012 & UK & 6 & 6 & - & 68 & 69 & - & $\mathrm{cCR}$ & प्राप्र & PNCT \\
\hline & 2004 & Brazil & 71 & 22 & - & 58.1 & 53.6 & - & $\mathrm{cCR}$ & वस्त $+\mathrm{CT}$ & PNCT \\
\hline & 2016 & Taiwan & 18 & 26 & - & 67.5 & 63.7 & - & $\mathrm{cCR}$ & प्रा & RNCT \\
\hline & 2015 & Korea & 8 & 28 & 16 & 64 & 70 & 70 & $\mathrm{cCR}$ & प्सा & PNCT \\
\hline & 2015 & China & 30 & 92 & - & 62 & 56 & - & $\mathrm{cCR}$ & प्राप्रा & PNCT \\
\hline & 2018 & Taiwan & 202 & 2542 & 36 & - & - & - & $\mathrm{cCR}$ & प्रापा & PNCT \\
\hline \multirow[t]{2}{*}{ 3] } & 2016 & Netherlands & 85 & - & 15 & 62.7 & - & 65.8 & cCR and near-cCR & 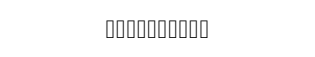 & RNCT \\
\hline & 2011 & Netherlands & 21 & 20 & - & 65 & 66 & - & cCR & प्राप्रा & PNCT \\
\hline \multirow[t]{4}{*}{ )] } & 2016 & UK & 129 & 109 & - & 66.9 & 65 & - & $\mathrm{cCR}$ & प्रापा & PNCT \\
\hline & 2012 & USA & 32 & 57 & - & 70 & 60 & - & $\mathrm{cCR}$ & 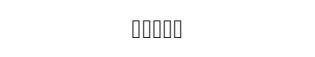 & PNCT \\
\hline & 2015 & USA & 18 & 30 & - & 62.3 & 60.4 & - & $\mathrm{cCR}$ & प्राप्रा & RNCT \\
\hline & 2019 & Korea & 15 & 129 & 25 & 74 & 64.8 & 73 & $\mathrm{cCR}$ & प्राप्रा & RNCT \\
\hline
\end{tabular}




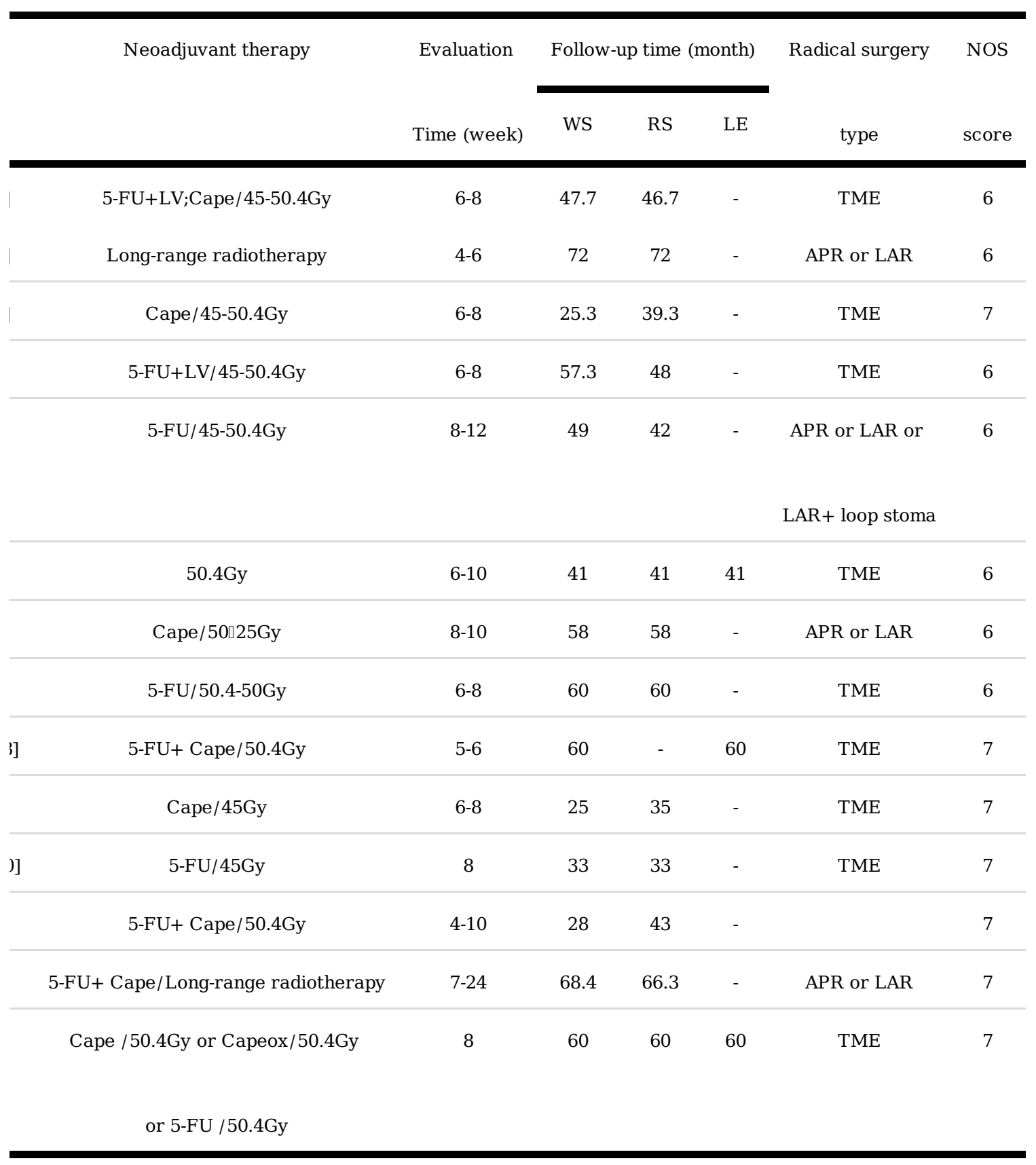




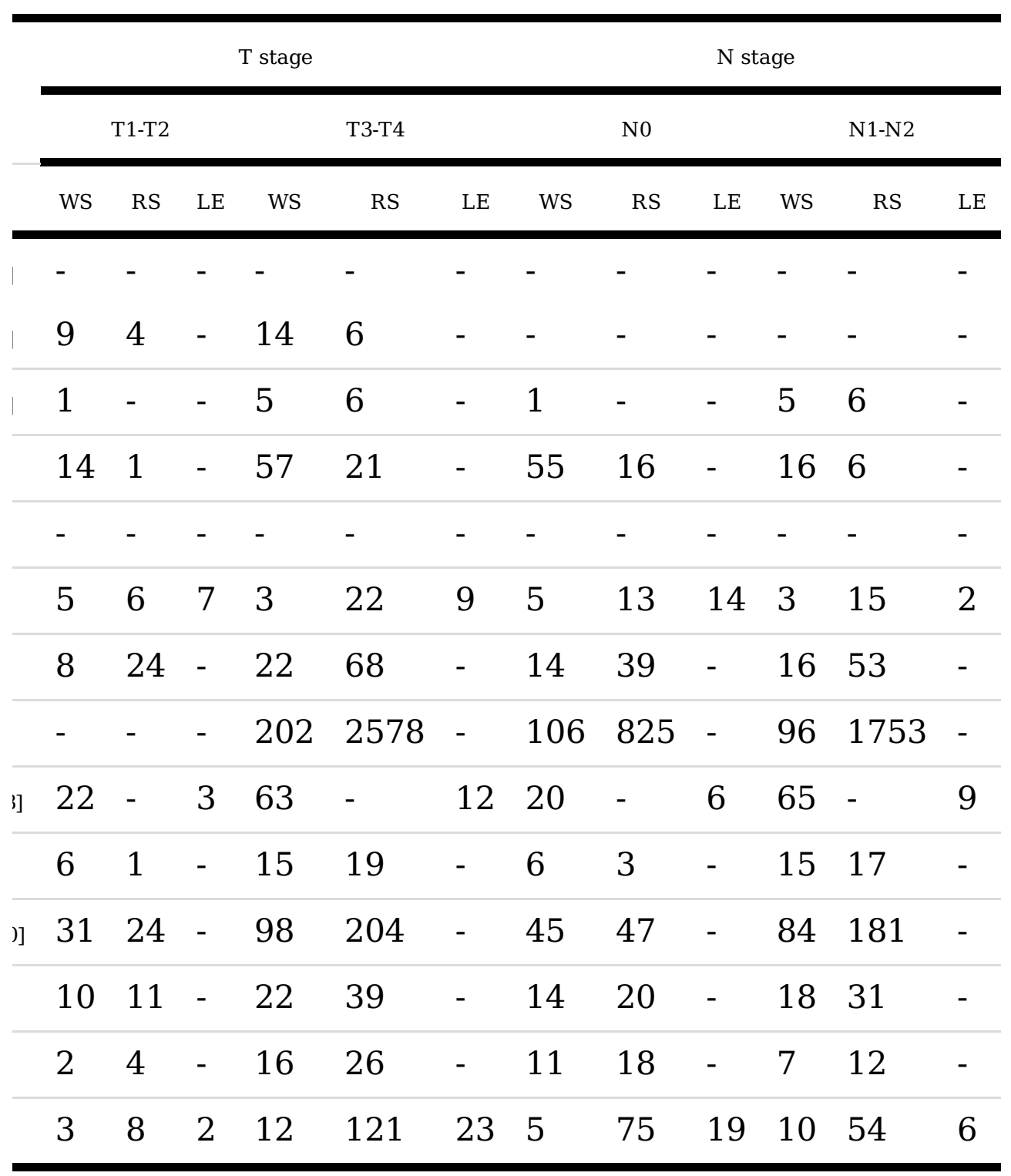




\begin{tabular}{|c|c|c|c|c|c|c|c|c|c|c|c|c|c|c|c|}
\hline & \multicolumn{12}{|c|}{ Clinical stage } & \multicolumn{3}{|c|}{ Quality control } \\
\hline & \multicolumn{3}{|c|}{$\square$} & \multicolumn{3}{|c|}{$\square$} & \multicolumn{3}{|c|}{$\square$} & \multicolumn{3}{|c|}{$\square$} & \multirow[t]{2}{*}{ Selection } & \multirow[t]{2}{*}{ comparability } & \multirow[t]{2}{*}{ outcome } \\
\hline & WS & RS & LE & wS & RS & LE & WS & RS & $\mathrm{LE}$ & ws & RS & LE & & & \\
\hline & 3 & 0 & - & 12 & 15 & - & 12 & 36 & - & 1 & 2 & - & 2 & 2 & 2 \\
\hline & - & - & - & - & - & - & - & - & - & - & - & - & 3 & 2 & 1 \\
\hline & - & - & - & - & - & - & - & - & - & - & - & - & 3 & 2 & 2 \\
\hline & - & - & - & - & - & - & - & - & - & - & - & - & 2 & 3 & 1 \\
\hline & - & - & - & 11 & 8 & & 7 & 18 & - & - & - & - & 2 & 2 & 2 \\
\hline & - & - & - & - & - & - & - & - & - & - & - & - & 3 & 2 & 1 \\
\hline & - & - & - & - & - & - & - & - & - & - & - & - & 3 & 1 & 2 \\
\hline & - & - & - & 107 & 828 & - & 95 & 1750 & - & - & - & - & 2 & 2 & 2 \\
\hline \multirow[t]{2}{*}{ 3] } & - & - & - & - & - & - & - & - & - & - & - & - & 3 & 3 & 1 \\
\hline & - & - & - & - & - & - & - & - & - & - & - & - & 3 & 2 & 2 \\
\hline \multirow[t]{4}{*}{ )] } & - & - & - & - & - & - & - & - & - & - & - & - & 2 & 3 & 2 \\
\hline & 8 & 2 & - & 6 & 18 & - & 18 & 31 & - & - & - & - & 3 & 3 & 1 \\
\hline & 1 & 2 & - & 10 & 16 & - & 6 & 12 & - & 1 & 0 & - & 2 & 3 & 2 \\
\hline & - & - & - & - & - & - & - & - & - & - & - & - & 2 & 3 & 2 \\
\hline
\end{tabular}




\begin{tabular}{|c|c|c|c|c|c|c|c|c|c|c|c|c|c|c|}
\hline \multirow[t]{3}{*}{ Study } & \multicolumn{10}{|c|}{ Pathlologic T stage } & \multicolumn{4}{|c|}{ Pathlologic N stage } \\
\hline & \multicolumn{2}{|c|}{ урт0 } & \multicolumn{2}{|c|}{ ypT1 } & \multicolumn{2}{|c|}{ урт2 } & \multicolumn{2}{|c|}{ урт3 } & \multicolumn{2}{|c|}{ урТ4 } & \multicolumn{2}{|c|}{ ypN0 } & \multicolumn{2}{|c|}{ ypN1-2 } \\
\hline & RS & LE & RS & LE & RS & LE & RS & LE & RS & LE & RS & LE & RS & LE \\
\hline Araujo[11] & - & - & - & - & - & - & - & - & - & - & - & - & - & - \\
\hline Ayloor[12] & 6 & - & - & - & 3 & - & 1 & - & - & - & 6 & - & 5 & - \\
\hline Dalton[13] & - & - & - & - & - & - & - & - & - & - & - & - & - & - \\
\hline Habr[6] & - & - & - & - & - & - & - & - & - & - & - & - & - & - \\
\hline Lai[14] & - & - & - & - & - & - & - & - & - & - & - & - & - & - \\
\hline Lee[15] & 13 & 6 & 2 & 6 & 9 & 4 & 4 & 0 & 0 & 0 & 24 & - & 4 & - \\
\hline Li[16] & - & & - & & - & - & & - & - & - & - & - & - & - \\
\hline $\operatorname{Lin}[17]$ & 370 & - & 118 & - & 573 & - & 1315 & - & 169 & - & 1704 & - & 826 & - \\
\hline Martens[18] & - & 9 & - & 1 & - & 5 & - & - & - & - & - & - & - & - \\
\hline Mass[19] & - & & - & & - & - & & - & - & - & - & - & - & - \\
\hline Renehan[20] & - & & - & & - & - & & - & - & - & - & - & - & - \\
\hline Smith[21] & - & & - & & - & - & & - & - & - & - & - & - & - \\
\hline Smith[22] & - & & - & & - & - & & - & - & - & - & - & - & - \\
\hline Yeom[23] & 46 & 12 & 47 & 11 & - & - & 36 & 2 & - & - & 108 & - & 21 & - \\
\hline
\end{tabular}

Notes: PNCT: prospective non-randomized controlled trial; RNCT: retrospective nonrandomized controlled trial; 5-FU:5-Fluorouracil; Cape: Capecitabine; RS: radical surgery; TME: total mesorectal excision; APR: abdomi-l-perineal resection; LAR: Low anterior resection.

Diagnostic criteria of cCR: $\square$ no residual tumor and white scar in endoscopy; $\square$ negative biopsies from the white scar; $\square$ no palpable tumor with digital rectal exam (DRE), $\square$ no suspicious lymph nodes in MRI $\square$ no residual tumor or residual fibrosis in MRI

Diagnostic criteria of near-cCR: \small residual erythematous ulcer or irregular wall

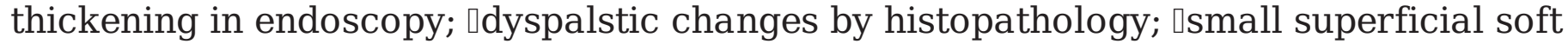
irregularity in digital rectal exam (DRE); ঢobvious downstaging of lymph nodes without 
malignant enhancement pattern(remaining nodes $\geq 5 \mathrm{~mm}$ ) in $\mathrm{MRI}$; $\square$ obvious downstaging with residual fibrosis but irregular aspect in MRI.

\section{Figures}

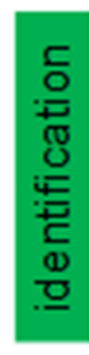

Total included records $(\mathrm{N}=7207)$

Pubmed( $\mathrm{N}=5617) ;$;Cochrane library( $\mathrm{N}=1158)$

Wanfang database $(\mathrm{N}=50) ; \mathrm{CNKI}$ database $(\mathrm{N}=382)$

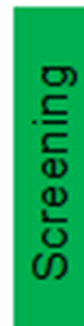

Duplicated records $(\mathrm{N}=5235)$

Title and abstract screened(N=1972)
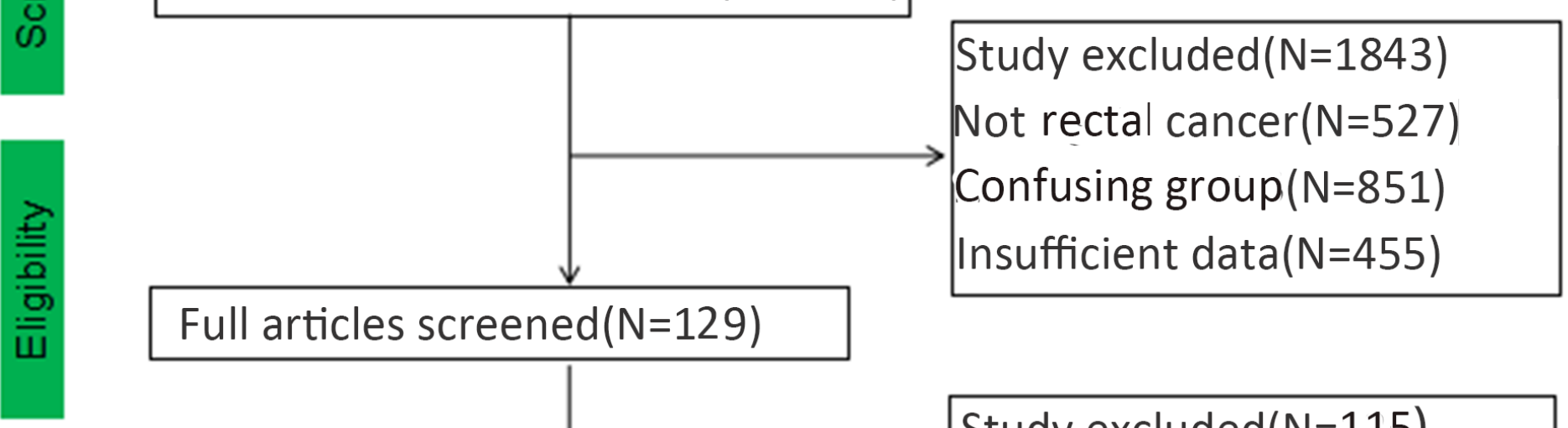

Full articles screened( $\mathrm{N}=129)$
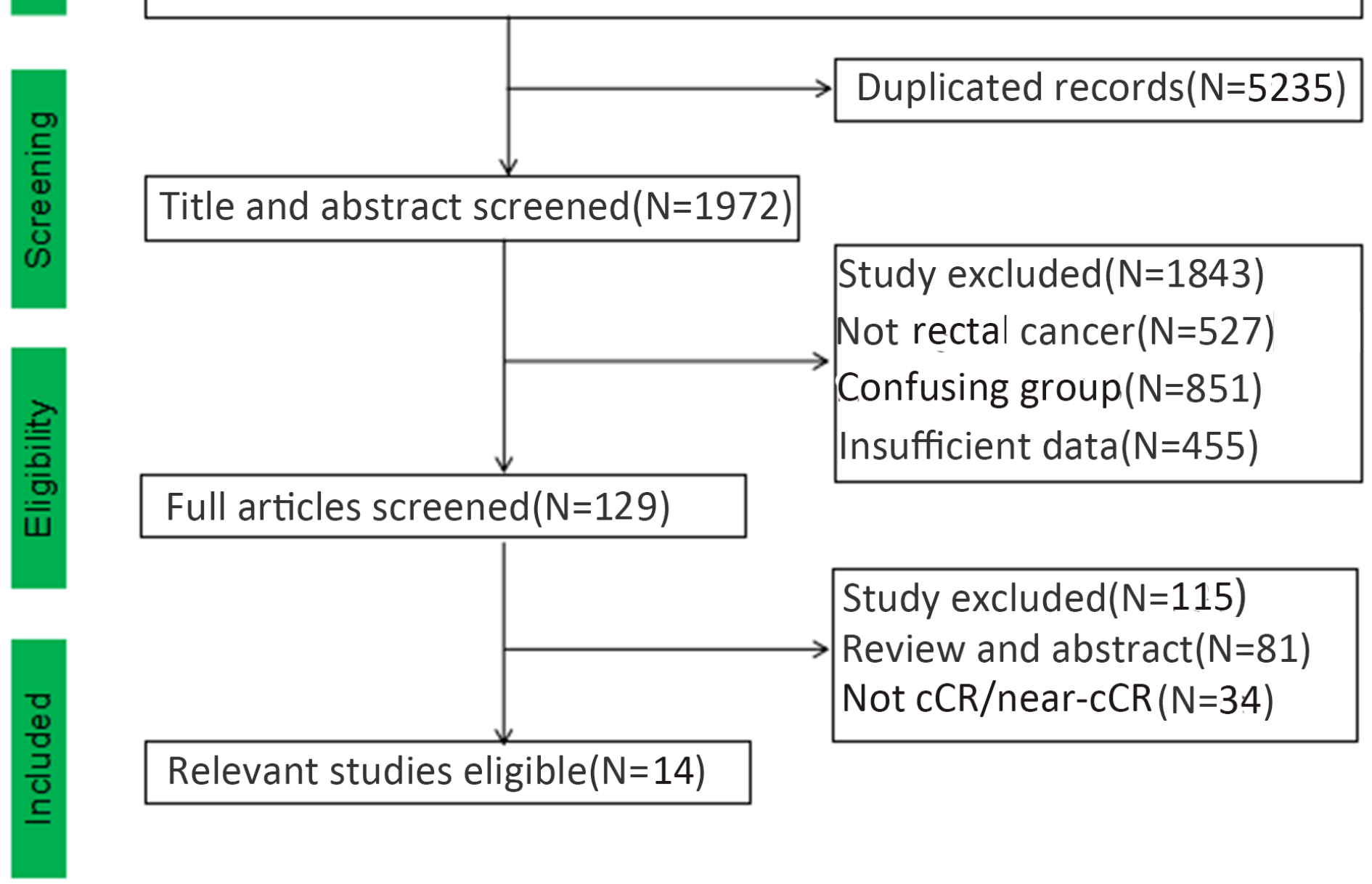

Relevant studies eligible( $\mathrm{N}=14)$

\section{Figure 1}

Flowchart of the included studies 


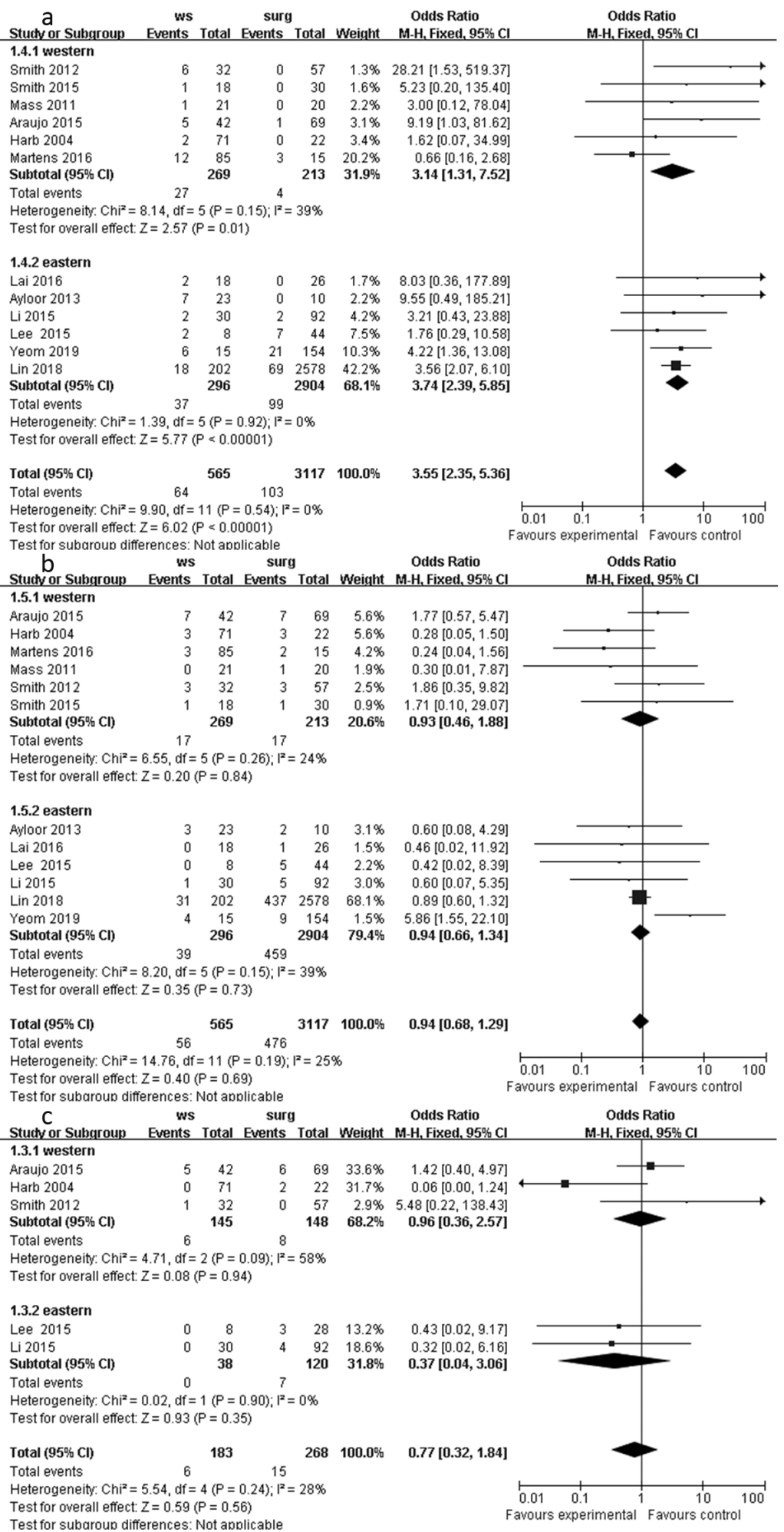

\section{Figure 2}

Outcomes of WS group versus Surgery group. a. Local recurrence; b: distant metastasis, c: cancer related death 


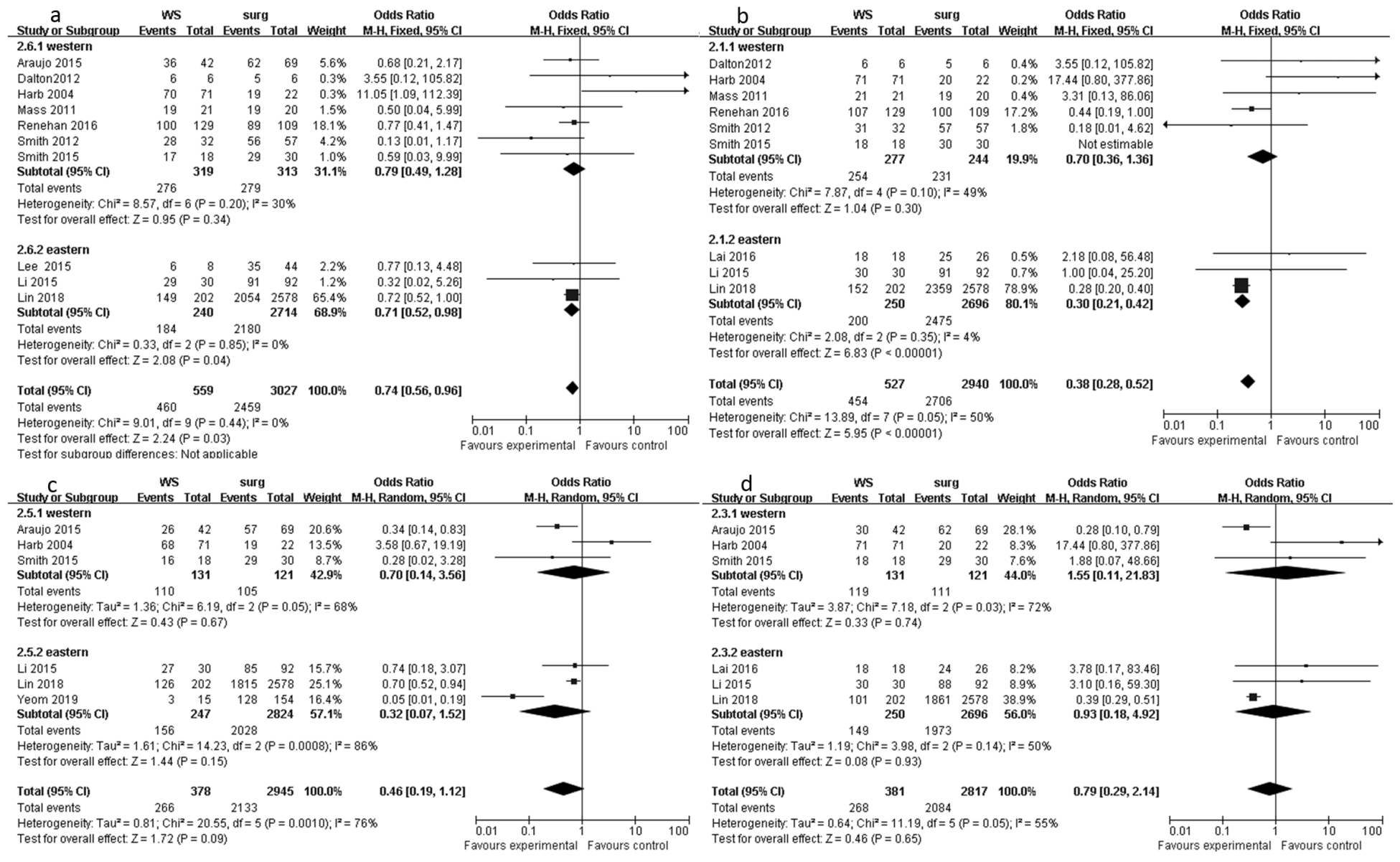

\section{Figure 3}

Outcomes of WS group versus Surgery group. a. 2-year DFS; b: 2-year OS; c: 5-year DFS; d: 5-year OS 


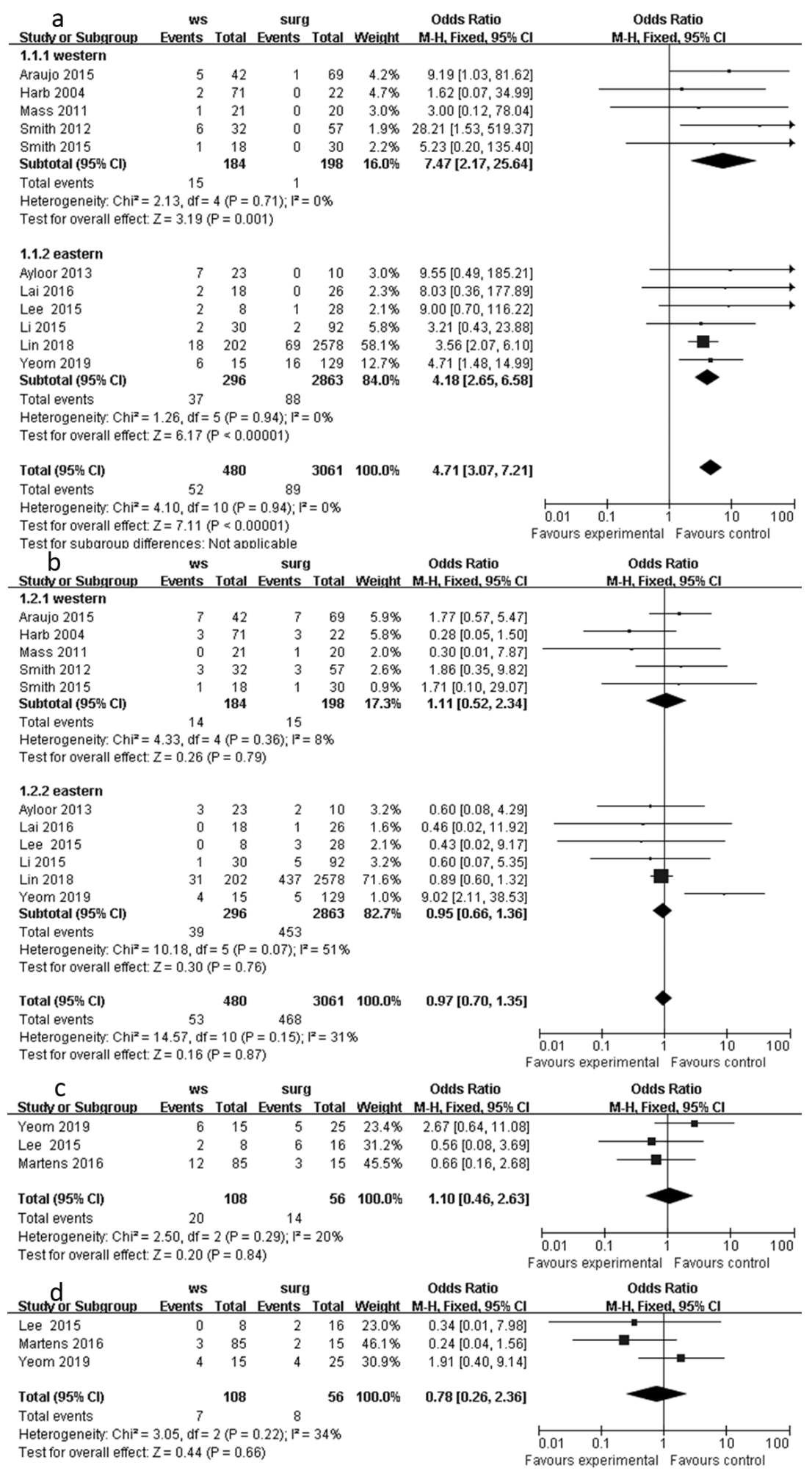

\section{Figure 4}

Outcomes of WS group versus Radical surgery group. a. Local recurrence; b: distant metastasis; Outcomes of WS group versus Local excision group. c. Local recurrence; d: distant metastasis; 


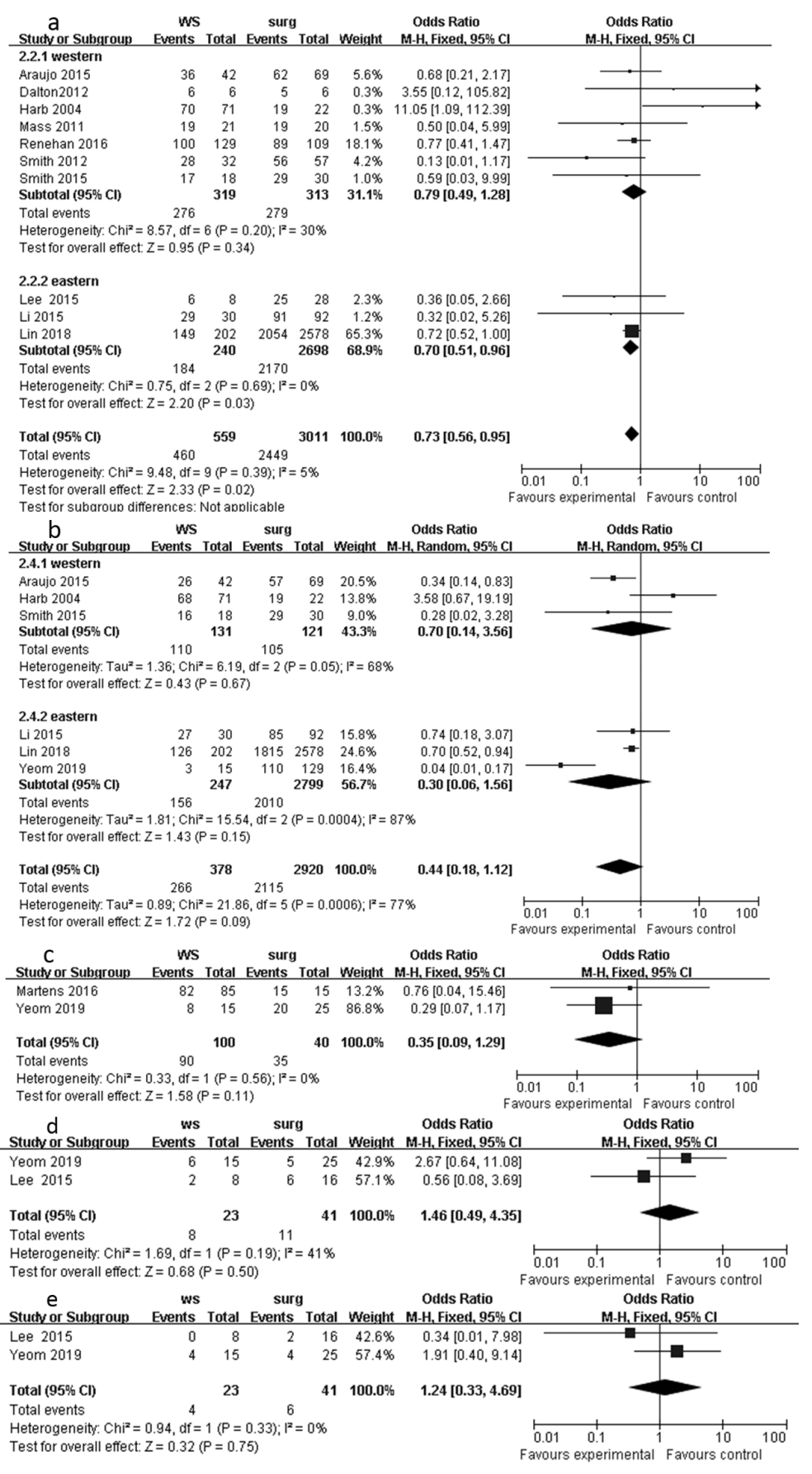

\section{Figure 5}

Outcomes of WS group versus Radical surgery group. A: 2-year DFS; b: 5-year DFS; Outcomes of WS group versus Local excision group. c: 3-year DFS; Outcomes of WS group versus local excision group(cCR). d:Local recurrence; e:Distant metastasis 
This is a list of supplementary files associated with this preprint. Click to download.

- additionalfile1.doc 